\title{
Supercurrent Spectroscopy of Andreev States
}

\author{
L. Bretheau, Ç. Ö. Girit, C. Urbina, D. Esteve, and H. Pothier* \\ Quantronics Group, Service de Physique de l'État Condensé (CNRS, URA 2464), \\ IRAMIS, CEA-Saclay, 91191 Gif-sur-Yvette, France \\ (Received 20 September 2013; published 27 December 2013)
}

\begin{abstract}
We measure the excitation spectrum of a superconducting atomic contact. In addition to the usual continuum above the superconducting gap, the single-particle excitation spectrum contains discrete, spindegenerate Andreev levels inside the gap. Quasiparticle excitations are induced by a broadband on-chip microwave source and detected by measuring changes in the supercurrent flowing through the atomic contact. Since microwave photons excite quasiparticles in pairs, two types of transitions are observed: Andreev transitions, which consist of putting two quasiparticles in an Andreev level, and transitions to odd states with a single quasiparticle in an Andreev level and the other one in the continuum. In contrast to absorption spectroscopy, supercurrent spectroscopy allows detection of long-lived odd states.
\end{abstract}

DOI: 10.1103/PhysRevX.3.041034

Subject Areas: Mesoscopics, Quantum Physics, Superconductivity

\section{INTRODUCTION}

The Josephson supercurrent between two superconductors [1] exists in all types of weak links, including tunnel junctions, constrictions, molecules, and normal metals. Weak links differ one from another by their quasiparticle excitation spectrum, which is determined by the length of the weak link and the transmission probabilities $\tau_{i}$ for electrons through each conduction channel $i$. In tunnel junctions, $\tau_{i} \ll 1$, and all excitations conserving electron parity require energies at least equal to $2 \Delta$, where $\Delta$ is the superconducting gap energy. With energy $2 \Delta$, a pair can be broken and two quasiparticles can be created at the gap energy $\Delta$. This is the same situation as in a bulk superconductor. In contrast, the excitation spectrum of weak links that have well-transmitted channels, such as superconducting constrictions, contains subgap spindegenerate Andreev levels (Andreev doublets) [see Fig. 1(a)]. The energy of the Andreev level associated with one channel with transmission $\tau$ in a short weak link is $E_{A}=\Delta \sqrt{1-\tau \sin ^{2}(\delta / 2)}$ [2,3], with $\delta$ the superconducting phase difference across the weak link. The lowest energy excitation that conserves electron parity, the "Andreev transition," has an energy $2 E_{A}$ : It consists in the creation of two quasiparticles in the Andreev level [red double arrow in Fig. 1(a)], which can be thought of as the excitation of a pair localized at the weak link $[4,5]$. We recently reported microwave spectroscopy of this Andreev transition in superconducting atomic contacts [4]. There is

\footnotetext{
*Corresponding author. hugues.pothier@cea.fr

Published by the American Physical Society under the terms of the Creative Commons Attribution 3.0 License. Further distribution of this work must maintain attribution to the author(s) and the published article's title, journal citation, and DOI.
}

a second type of excitation, with energy at least $E_{A}+\Delta$, in which a localized Andreev pair is broken into one quasiparticle in the Andreev level and one in the continuum [green arrows in Fig. 1(a)]. This process was addressed theoretically in recent works [6-8] but has never been observed experimentally. Here, we describe how "supercurrent spectroscopy" reveals all possible transitions involving Andreev states (Fig. 1). This method is based on measuring the supercurrent through a weak link and detecting changes induced by microwave excitation [9].

The supercurrent in a weak link results from the phase dependence of its energy, which depends on the occupation of the Andreev levels. In the case of a single channel [see Fig. 1(b)], the ground state, with energy $-E_{A}$, carries a supercurrent $-I_{A}=-\varphi_{0}^{-1}\left(\partial E_{A} / \partial \delta\right)$. The energy of an odd state, with a single Andreev excitation, is zero: It carries no supercurrent. The excited pair state with two Andreev excitations has energy $+E_{A}$ and therefore carries a supercurrent $+I_{A}$. This difference in the supercurrent associated with 0,1 , or 2 Andreev excitations is the elementary phenomenon needed to understand microwave- or voltage-induced variations of the supercurrent [10,11], as well as the current response to an ac field [12] in diffusive superconductor-normal-metal-superconductor junctions [13]. Measurements of the phase dependence of the supercurrent through atomic contacts with a few conduction channels revealed the spontaneous excitation to odd states and allowed characterization of their dynamics [14]. Here, we use the dependence of the supercurrent on the occupation of the Andreev doublet to reveal the complete excitation spectrum of an elementary, generic weak link: an atomic contact.

\section{EXPERIMENTAL SETUP}

The experimental setup is shown schematically in Fig. 2(a). An atomic contact is obtained by breaking in a 
(a)

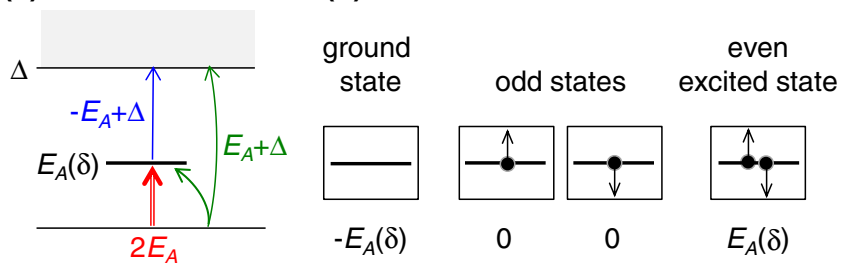

FIG. 1. (a) The single particle excitation spectrum for each channel of a weak link consists of a doubly degenerate Andreev level at energy $E_{A}(\delta)$ and a continuum of states at energies larger than the superconducting gap $\Delta$. Arrows indicate transitions that can be induced by microwaves. The four possible occupations of the Andreev level are shown in (b): They correspond to the ground state, the two odd states, and the excited pair state, with energies $-E_{A}, 0$, and $E_{A}$.

controlled manner a suspended constriction in an $\mathrm{Al}$ film $(\Delta=180 \mu \mathrm{eV})[5,15]$. It is placed in parallel with a tunnel Josephson junction having a much larger critical current $I_{0} \simeq 1.06 \mu \mathrm{A} \approx 20\left|I_{A}\right|$, hence forming an asymmetric SQUID. The number of conduction channels in the atomic contact and their transmission probabilities are determined from a fit of the current-voltage characteristic of the SQUID [16]. The magnetic flux $\phi$ through the SQUID loop fixes the phase difference at the atomic contact to $\delta=$ $\varphi+\gamma$, with $\varphi=2 \pi \phi / \phi_{0}$ the reduced flux, $\phi_{0}=h / 2 e$ the flux quantum, and $\gamma$ the phase across the SQUID junction. The SQUID is asymmetric enough so that $\gamma \simeq \arcsin \left(I_{b} / I_{0}\right)$ is determined only by the bias current $I_{b}$. The SQUID is capacitively coupled on chip to a small Josephson junction (critical current $48 \mathrm{nA}$ ), called the "emitter." Figure 2(b) shows a micrograph of the SQUID and the emitter. The emitter is biased at a voltage $V_{J}$ and, because of the ac Josephson effect, acts as a broadband microwave source at frequency $\nu_{J}=2 e V_{J} / h$. When $h \nu_{J}$ matches a transition energy in the atomic contact, a photon can be absorbed, and the occupation of Andreev states is modified. In "absorption spectroscopy" experiments performed on the same device [4], energy dissipated during excitation of an Andreev transition in the superconducting atomic contact was detected by measuring the dc current through the emitter. Here, instead, the corresponding change in the supercurrent $I_{\mathrm{AC}}(\delta)$ of the atomic contact is accessed by measuring the critical current of the SQUID: To a good approximation, the critical current of such an
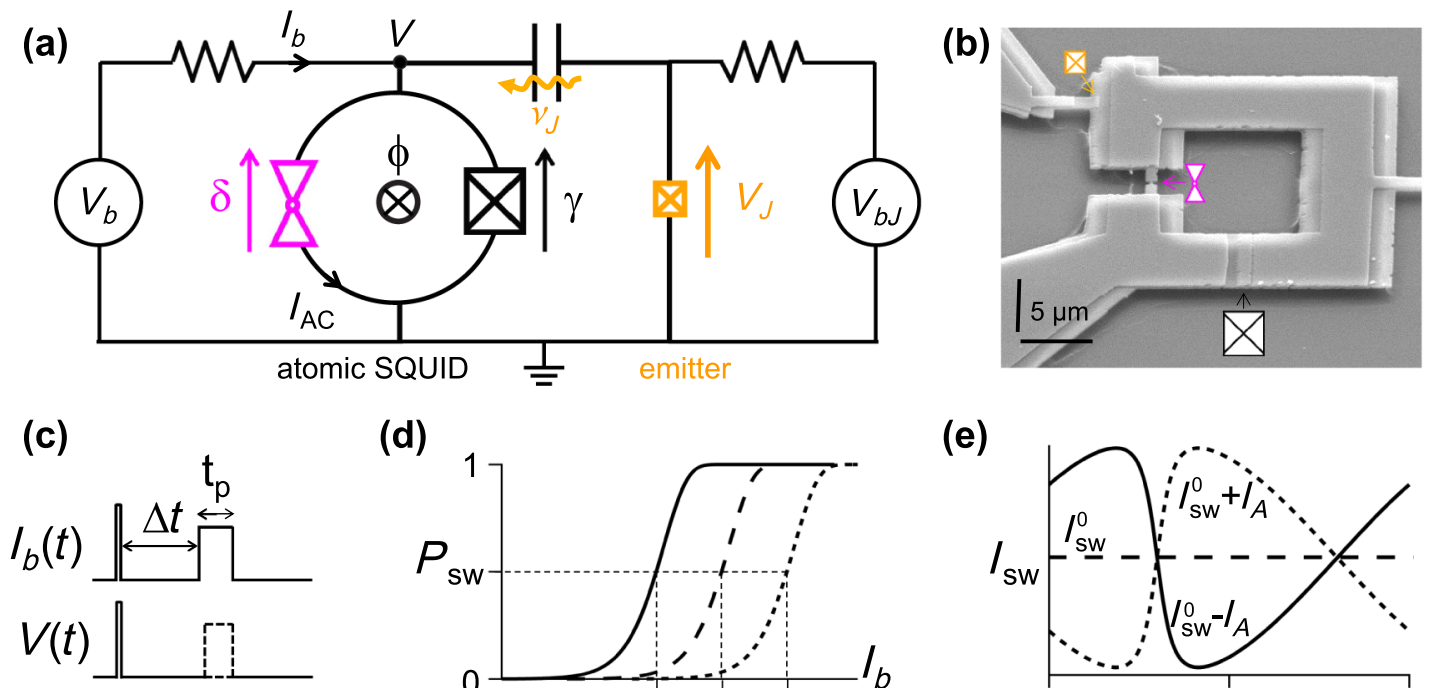

(d)

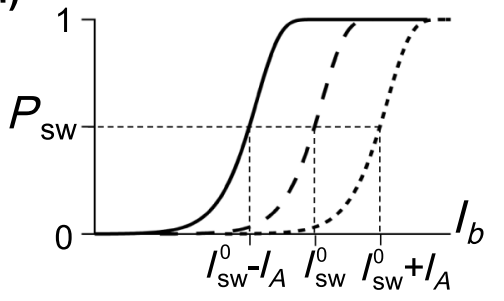

(e)

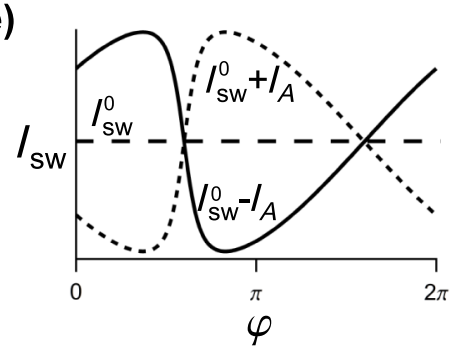

FIG. 2. (a) Simplified schematic of the experimental setup. An asymmetric SQUID is formed by an atomic contact (magenta triangles) and an ancillary Josephson junction (critical current $I_{0} \simeq 1.06 \mu \mathrm{A}, 20$ times larger than the typical critical current of a oneatom aluminum contact). In the absence of SQUID bias current $\left(I_{b}=0\right)$, the magnetic flux $\phi$ threading the loop imposes a phase $\delta \simeq \varphi \equiv 2 \pi \phi / \phi_{0}$ across the contact and determines its excitation spectrum and the loop current $I_{\mathrm{AC}}$. It is coupled through a capacitor to a voltage-biased Josephson junction (yellow checked box, critical current $48 \mathrm{nA}$ ) used as an emitter: When biased at voltage $V_{J}$, it produces an ac current at Josephson frequency $\nu_{J}=2 e V_{J} / h$. (b) Micrograph of the sample, seen under an angle (scale bars indicate $5 \mu \mathrm{m}$ in two directions). (c) The SQUID switching current is measured by applying sequences of current $\left(I_{b}\right)$ pulses of duration $t_{p}$. At time $\Delta t$ before each measurement pulse, any memory of the response to the previous pulse is erased by a strong current pulse ("prepulse") that forces the SQUID to switch [14]. Switching events are detected by the appearance of a voltage $V$ across the SQUID. (d) Sketch of the switching probability $P_{\mathrm{sw}}$ of the atomic SQUID as a function of the bias current $I_{b}$, for the atomic contact in its ground state (solid line), in an odd state (dashed line), and in the excited pair state (short-dashed line). For this figure, we assumed a single channel and $I_{A}>0$. The switching current of the SQUID Josephson junction in the absence of an atomic contact is $I_{\text {sw }}^{0}$. (e) Sketch of the switching current of the SQUID as a function of the phase $\varphi$ in the ground, odd, or excited even states. 
asymmetric SQUID is the sum of the critical current $I_{0}$ of the SQUID Josephson junction and of $I_{\mathrm{AC}}(\delta)$.

In practice, because of thermal fluctuations, the SQUID switches to a finite voltage state at a current inferior to the critical current. The current $I_{\mathrm{AC}}(\delta)$ is therefore inferred from the switching probability $P_{\mathrm{sw}}\left(I_{b}\right)$ when a bias current pulse of height $I_{b}$ and duration $t_{p}=1 \mu \mathrm{s}$ is applied [Fig. 2(c)] [17]. The probability $P_{\mathrm{sw}}\left(I_{b}\right)$ increases smoothly from 0 to 1 around the switching current, which is $I_{\mathrm{sw}}^{0}$ for the junction alone and $\sim I_{\mathrm{sw}}^{0}+I_{\mathrm{AC}}(\delta)$ for the atomic SQUID [16], i.e., $I_{\mathrm{sw}}^{0}-I_{A}$ in the ground state, $I_{\mathrm{sw}}^{0}+I_{A}$ in the excited state, and $I_{\mathrm{sw}}^{0}$ in the odd states [Figs. 2(d) and 2(e)]. The principle of our experiment is to measure changes of $P_{\mathrm{sw}}$ induced by the microwave excitation.

The switching probability is calculated from the response to a train of $10^{4}$ pulses. For each value of the flux $\phi$, the height of the measurement pulse is set to $I_{\mathrm{sw}}$ such that $P_{\mathrm{sw}}=0.5$ in the absence of microwaves, i.e., at $V_{J}=0$. Assuming that the atomic contact is then in its ground state, $I_{\mathrm{sw}}=I_{\mathrm{sw}}^{0}-I_{A}(\delta)$ [Figs. 2(d) and 2(e)]. Then $P_{\text {sw }}$ is recorded as a function of the microwave excitation frequency set by $V_{J}$. This procedure is repeated for all values of $\phi$, and one obtains a map of $P_{\mathrm{sw}}$ as a function of $\varphi$ and $V_{J}$ : the "switching spectrum." Such a spectrum is shown in Fig. 3(a) for a contact with two channels of significant transmissions: $\tau_{1}=0.985, \tau_{2}=0.37$. Most of the features of the rich spectrum, which has numerous phase- and energy-dependent lines and plateaus, are explained below and in Ref. [18].

\section{INTERPRETATION OF THE SPECTRUM}

We first discuss general aspects of the spectrum that are unrelated to Andreev physics. The white band slightly above $2 e V_{J} \simeq 0.5 \Delta$ with no data corresponds to voltages that cannot be accessed because of an instability in the I-V of the emitter when the Josephson frequency matches the plasma frequency $\nu_{p}$ of the SQUID [4]. When $2 e V_{J}>$ $h \nu_{p}$, the background of the image is light blue, corresponding to an overall increase of the switching probability to $P_{\mathrm{sw}} \simeq 0.55$, which is interpreted as an increased noise temperature when the emitter is biased. When $2 e V_{J}<h \nu_{p}$, the overall increase in $P_{\mathrm{sw}}$ is even stronger $\left(P_{\mathrm{sw}} \simeq 0.6-0.8\right)$, with broad, phase-independent stripes in the spectrum, corresponding to a $10-20-\mathrm{mK}$ increase in the
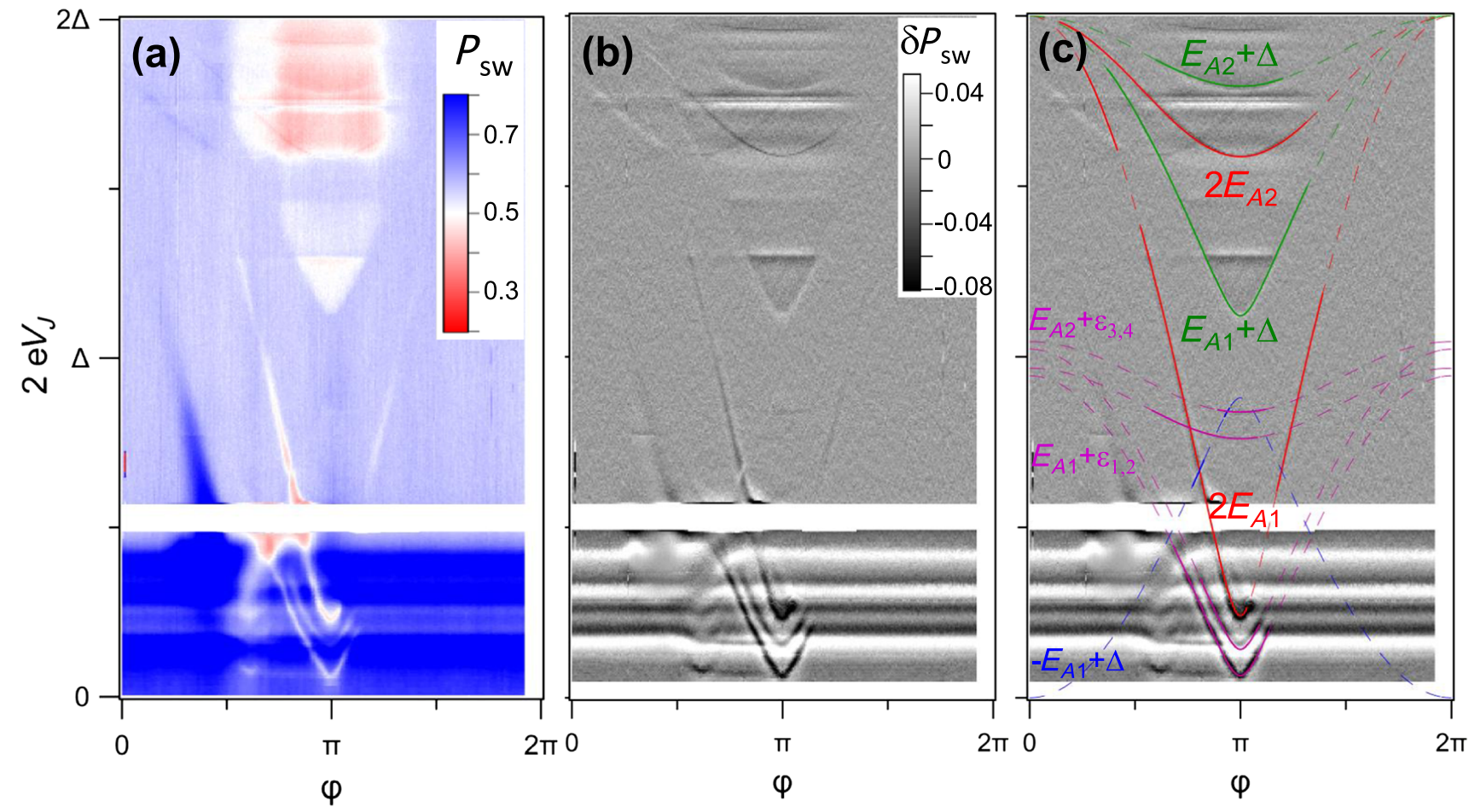

FIG. 3. (a) Switching spectrum $P_{\mathrm{sw}}\left(\varphi, 2 e V_{J}\right)$ measured on an atomic contact with two channels of transmissions 0.985 and 0.37 . (b) Filtered spectrum $\delta P_{\mathrm{sw}}\left(\varphi, 2 e V_{J}\right)$ (see text) highlighting rapid variations of the switching probability with energy. (c) Same as (b), with transitions underlined. Dashed lines correspond to the predicted positions of transitions, and solid lines underline where they are actually visible in the data. Colors correspond to those of the arrows in Fig. 1(a). Red lines: Andreev transitions at $2 e V_{J}=2 E_{A 1,2}$. Green lines: Threshold $2 e V_{J}=E_{A 1,2}+\Delta$ for transitions odd states with one quasiparticle in an Andreev level and another one in the continuum. Blue line: Threshold $2 e V_{J}=-E_{A 1}+\Delta$ for transitions from odd states to even states by transferring a quasiparticle from the Andreev level into the continuum. Magenta lines: Andreev transitions induced by the second harmonic of the excitation when $2 e V_{J}=E_{A 1,2}$, shifted by energies $\varepsilon_{1-4}=-5,2,-3$, and $4 \mu \mathrm{eV}$ (from bottom to top). 
effective temperature, an effect attributed to resonant activation during the measurement pulse $[9,19]$.

\section{A. Identification of the transition lines}

Relevant to the physics of Andreev levels are changes in $P_{\text {sw }}$ that depend on the phase across the atomic contact. They are better seen in Fig. 3(b), where the slow components of $P_{\mathrm{sw}}\left(V_{J}\right)$ in Fig. 3(a) have been filtered out to obtain $\delta P_{\mathrm{sw}}$ (the signal was first smoothed on $0.17 \Delta$ intervals; the result was then subtracted from the original spectrum). Three types of transitions are resolved, corresponding to the arrows in Fig. 1(a): the Andreev transition, i.e., the excitation of the Andreev pair (red arrow); the transition to an odd state with a single quasiparticle in the Andreev doublet, the second one being excited to the continuum (green arrows); the excitation of a quasiparticle from the Andreev level to the continuum (blue arrow).

Andreev transitions at $2 e V_{J}=2 E_{A}$ [red arrow in Fig. 1(a)] are seen as sharp V-shaped lines centered at $\varphi=\pi$, with minima at $2 E_{A 1}(\pi) \simeq 0.25 \Delta$ for the channel with transmission $\tau_{1}=0.985$ and $2 E_{A 2}(\pi) \simeq 1.6 \Delta$ for the channel with transmission $\tau_{1}=0.37$ [red lines in Fig. 3(c)]. The variations across the spectra of the intensity of the lines are discussed in Ref. [18].

There are, in addition, two strong lines parallel to $E_{A 1}(\delta)$ and two faint lines parallel to $E_{A 2}(\delta)$ in the spectrum [magenta lines in Fig. 3(c)]. They correspond to exciting Andreev transitions with the second harmonic of the Josephson frequency. We do not understand, however, why the lines are shifted up or down with respect to the expected position $2 e V_{J}=E_{A i}$ by a few $\mu \mathrm{V}$.

Transitions from the ground state to an odd state with one quasiparticle in the Andreev doublet (energy $E_{A}$ ) and another one at energy larger than $\Delta$ in the continuum [green arrows in Fig. 1(a) and green lines in Fig. 3(c)] are best seen in the first channel as a reduced $P_{\text {sw }}$ [white in Fig. 3(a)] in a region defined by $2 e V_{J}>E_{A 1}+\Delta$. The corresponding threshold for the second channel is also seen at $2 e V_{J}=E_{A 2}+\Delta$.

There is a faint transition at $2 e V_{J}=-E_{A 1}+\Delta$ [blue line in Fig. 3(c)]. It corresponds to exciting a quasiparticle from the Andreev doublet of the first channel to the continuum [blue arrow in Fig. 1(a)]. The detection of this odd-even transition is explained if one assumes a finite probability that the doublet is occupied in the absence of excitation (as already found in former experiments [14]).

Blurred replica of the transition lines are visible shifted leftwards by about $0.4 \pi$. They correspond to transitions induced not before but during the measurement pulse, as the microwave produced by the emitter is applied continuously. During the pulse, a finite current flows through the SQUID junction, and the phase across the contact is no longer $\delta=\varphi$ but $\delta=\varphi+\gamma_{\mathrm{sw}}$ with $\gamma_{\mathrm{sw}} \simeq$ $\arcsin \left(I_{\mathrm{sw}}^{0} / I_{0}\right) \sim 0.4 \pi$. The replica of the oddeven transition discussed in the previous paragraph is responsible for the sharp disappearance of the Andreev transition line at $\varphi \gtrsim 1.04 \pi$, as discussed in Ref. [18].

\section{B. Sign of changes in $\boldsymbol{P}_{\mathrm{sw}}$}

In most of the spectrum $(0.6 \pi<\varphi \lesssim 1.5 \pi)$, the Andreev transitions and the transitions to odd states manifest themselves by a decrease of the switching probability $P_{\text {sw }}$ [white on blue background in Fig. 3(a), black on grey background in Fig. 3(b)]. In contrast, the faint odd-even transition gives an increased $P_{\mathrm{sw}}$. At $\varphi \sim 0.6 \pi$, all transitions disappear and then reappear at $\varphi<0.6 \pi$ with opposite sign of the change in $P_{\mathrm{sw}}$ (this effect is particularly visible on the line at $2 e V_{J}=2 E_{A 2}$ and on its replica).

The explanation is found in Figs. 2(d) and 2(e): Starting from the ground state (solid lines), all transitions lead to a decrease of $P_{\mathrm{sw}}$ in the regions where $I_{A}(\delta)>0$. Since $\delta=\varphi+\gamma_{\mathrm{sw}}$ during the measurement pulse, $I_{A}(\delta)>0$ when $0.6 \pi<\varphi<1.6 \pi$.

The odd-even transition (blue line) is seen because of a finite probability to initially find the Andreev level in an odd state. Then, the initial pulse height, set such that $P_{\text {sw }}=0.5$ when $V_{J}=0$, corresponds to a current between $I_{\mathrm{sw}}^{0}-I_{A}$ and $I_{\mathrm{sw}}^{0}$, and transitions to the ground state cause an increase in $P_{\mathrm{sw}}$.

\section{Dynamics of the Andreev-level occupation during spectroscopy}

We now analyze more in depth the amplitude of changes in $P_{\mathrm{sw}}$ and the nature of the states that are detected in the experiment. When exciting Andreev transitions, one expects that $I_{\mathrm{AC}}(\delta)$ changes by $2 I_{A}$ because the supercurrent carried by a channel of transmission $\tau$ changes from $-I_{A}$ to $+I_{A}$. This turns out not to be the case: Setting $\varphi$ and $V_{J}$ on the Andreev transition line $2 e V_{J}=$ $2 E_{A 1}$, the curve $P_{\mathrm{sw}}\left(I_{b}\right)$ does not show the step corresponding to the excited state (see Fig. 4). An explanation is sketched in Fig. 5: When the Andreev pair is excited, it often decays to its ground state; cycles of excitation and relaxation give rise to a dc current through the emitter [4]. But it can also decay to an odd state ("quasiparticle poisoning"), which is long-lived [14]. Just before the measurement pulse, the probability to be in an odd state can therefore be large. When the current increases through the SQUID, the phase across the contact changes by up to $\gamma_{\mathrm{sw}}$, the Andreev energy changes from $E_{A}(\varphi)$ to $E_{A}(\varphi+$ $\left.\gamma_{\mathrm{sw}}\right)$, and the excitation is no longer resonant with the Andreev transition. The population of the excited state then decays at a rate $\Gamma_{r}$. If $\Gamma_{r}>t_{r}^{-1}\left(\Gamma_{r}>10 \mathrm{MHz}\right)$, with $t_{r} \simeq 0.1 \mu \mathrm{s}$ the rise time of the measurement pulse, it relaxes before the pulse has fully developed, and no change is detected in $P_{\mathrm{sw}}$ [22]. In contrast, the odd state is observed because its decay rate is, in general, much smaller [14,21]. Hence, quasiparticle poisoning acts as a "sample and hold" mechanism for detecting Andreev 


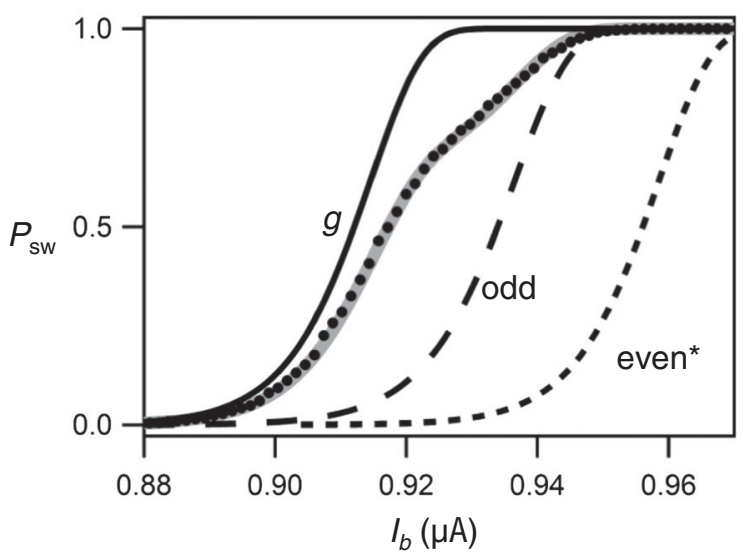

FIG. 4. Dots: Switching probability $P_{\mathrm{sw}}\left(I_{b}\right)$ measured near the minimum of the Andreev transition line at $2 E_{A 1}(2 \mathrm{eV} J=$ $0.24 \Delta, \varphi=1.02 \pi)$. Solid line: Calculated $P_{\mathrm{sw}}\left(I_{b}\right)$ with the two channels in the ground state (corresponding to a total current in the SQUID junction $I_{b}-I_{A 1}-I_{A 2}$ ). Dashed line: Calculated $P_{\text {sw }}\left(I_{b}\right)$ with the first channel in an odd state (corresponding to a total current in the SQUID junction $I_{b}-I_{A 2}$ ). Grey curve: Fit of the data with sum of the two previous curves with weights 0.63 and 0.37. Short-dashed line: Calculated $P_{\mathrm{sw}}\left(I_{b}\right)$ with the first channel in the even excited state (corresponding to a total current in the SQUID junction $I_{b}+I_{A 1}-I_{A 2}$ ). A finite probability to find the channel in the even excited state would have resulted in a contribution of the short-dashed line to the data.

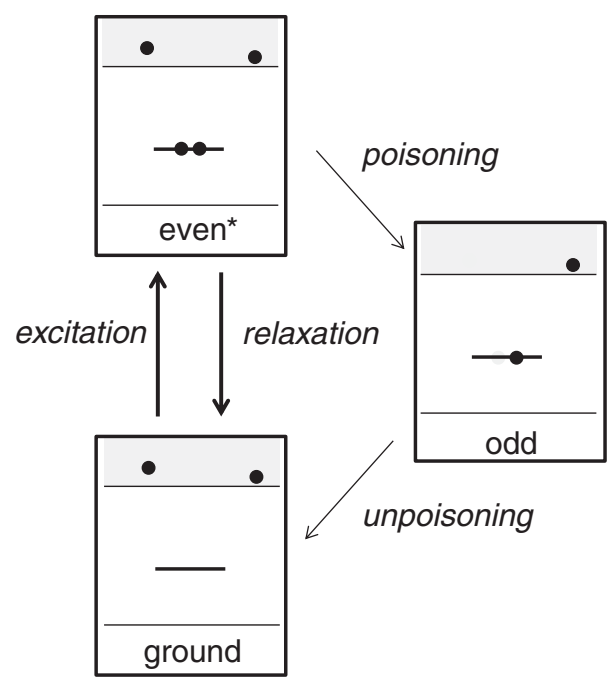

FIG. 5. Dynamics of Andreev-level occupation in the presence of a resonant excitation at frequency $2 E_{A}$. Andreev excitations are induced from the ground state (bottom) to the excited even state (even*, top). When followed by a direct relaxation, the process can immediately repeat itself and give rise to a dc current through the emitter junction, which is the signal used for absorption spectroscopy [4]. Alternatively, one quasiparticle in the Andreev level can recombine with a quasiparticle in the continuum and lead to an odd state (poisoning). Such states are longlived, allowing the detection of the preceding Andreev transition by supercurrent spectroscopy. Odd states relax to the ground state (unpoisoning) by a similar recombination process [20]. transitions. Note that transitions at energies greater than $E_{A i}+\Delta$ [green in Figs. 1(a) and 3(c)] leave the Andreev doublet directly in an odd state, and their observation does not require quasiparticle poisoning.

For phases such that $\Delta-E_{A 1}\left(\varphi+\gamma_{s w}\right)<2 E_{A 1}(\varphi)$, a new process comes into play: unpoisoning by the microwaves during the measurement pulse. Because the Andreev energy approaches $\Delta$ because of the phase shift $\gamma_{\mathrm{sw}}$, photons at energy $2 E_{A 1}(\varphi)$ can excite a quasiparticle from the Andreev level to the continuum during the measurement pulse. This process empties the Andreev level from an odd state and leads to the abrupt disappearance of the Andreev transition line at $\varphi \gtrsim 1.04 \pi$ (see Fig. 8 in Ref. [18]).

When the Andreev doublet is in an odd configuration, energy absorption at $2 E_{A}$ is hindered, and the dc current through the emitter associated with the absorption by the Andreev transition is suppressed. This was probed in time-resolved measurements of the emitter current. Quasiparticle poisoning and unpoisoning are then observed in real time as a telegraphic noise in the current, with time scales in the tens of ms (see Fig. 10 in Ref. [18]).

\section{COMPARISON OF ABSORPTION SPECTROSCOPY AND SUPERCURRENT SPECTROSCOPY}

Finally, we compare in Fig. 6 the absorption spectrum [4] and the switching spectrum measured on the same atomic contact. The spectra are different because absorption spectroscopy detects excitation and relaxation cycles that repeat themselves rapidly enough to give a substantial current through the emitter, whereas supercurrent spectroscopy requires long-lived states that do not decay during the measurement pulse rise time. Nevertheless, as explained above, transitions to the short-lived even excited states are also seen in supercurrent spectroscopy thanks to quasiparticle poisoning.

The switching spectrum contains more information, in particular, in the upper half of the spectrum. Remarkably, the Andreev transition in the second channel $\left(2 e V_{J}=2 E_{A 2}\right)$, which was barely visible in the absorption spectrum, is sharply resolved. The switching spectrum does not include the lines associated with the plasma mode of the SQUID at $2 e V_{J}=h \nu_{p}, 2 h \nu_{p}$ and $h \nu_{p}+2 E_{A 1}$ [see Fig. 6(c)], which are apparent in the absorption spectrum. The reason we do not see the two first is that the lifetime of the plasma mode, estimated to be in the ns range from the quality factor $Q \simeq 22$ of the corresponding peak in the I-V of the emitter, is much shorter than the rise time $t_{r}$ of the measurement pulse. However, one would expect to detect the third transition, at $2 e V_{J}=h \nu_{p}+2 E_{A 1}$, which corresponds to a simultaneous excitation of the plasma mode and of the Andreev doublet. We speculate that even if poisoning occurs in the same manner as when $2 e V_{J}=2 E_{A 1}$, photons at energy $h \nu_{p}+2 E_{A 1}$ trigger unpoisoning (see 

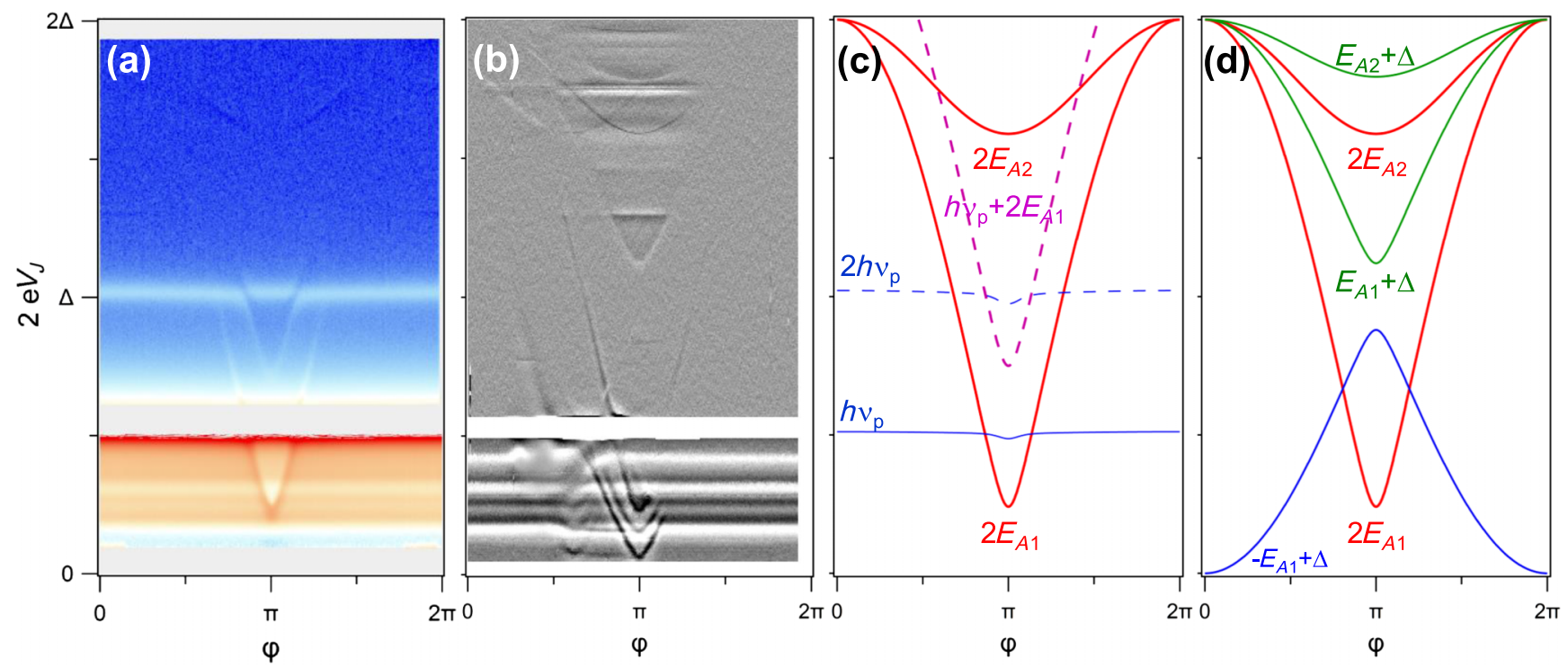

FIG. 6. Comparison of the absorption spectrum (a) and of the switching spectrum (b) taken on the same contact $\left(\tau_{1}=0.985, \tau_{2}=\right.$ 0.37). Panels (c) and (d) indicate the main transitions visible in (a) and (b), respectively. Solid red lines in (c, d): Andreev transitions at $2 e V_{J}=2 E_{A 1,2}$. Solid and dashed blue lines in (c): plasma transition, first and second harmonic $\left(2 e V_{J}=h \nu_{p}, 2 h \nu_{p}\right)$. Dashed magenta line in (c): Simultaneous excitation of Andreev and plasma modes $\left(2 e V_{J}=2 E_{A 1}+h \nu_{p}\right)$. We also show in (d), with green lines, the threshold $2 e V_{J}=E_{A 1,2}+\Delta$ for simultaneous excitations of quasiparticles in the Andreev doublet and in the continuum. Blue line in (d): Threshold $2 e V_{J}=-E_{A 1}+\Delta$ for transitions from odd states to even states having one quasiparticle in the continuum.

Ref. [18]) and the doublet is found only in its ground state [23].

\section{CONCLUSIONS}

The experimental results show that spectroscopy based on the measurement of the Josephson supercurrent allows one to detect single quasiparticle excitations in superconducting weak links. The entire excitation spectrum is explained by the presence of Andreev doublets that can be occupied by 0,1 , or 2 quasiparticles. Andreev transitions are detected when followed by quasiparticle poisoning, which acts as a "sample and hold" mechanism by placing the Andreev doublet in a long-lived odd state. We also demonstrate, for the first time, the possibility to induce, without injecting any charge [24], transitions from the (even) ground state to an odd state with a single excitation in the Andreev doublet (the second one being in the continuum). This type of transition could be used to prepare spin qubits based on odd states [25,26].

\section{ACKNOWLEDGMENTS}

We thank Alfredo Levy Yeyati, John Martinis, and Eva Dupont-Ferrier for discussions. We gratefully acknowledge help from other members of the Quantronics group, in particular, P. Senat and P. F. Orfila for invaluable technical assistance. This work was partially financed by ANR through projects DOC-FLUC, MASH, and by CNANO-Ile-de-France. The research leading to these results has received funding from the People Programme
(Marie-Curie Actions) of the European Union's Seventh Framework Programme (No. FP7/2007-2013) under REA Grant Agreement No. PIIF-GA-2011-298415. L. Bretheau and Ç. Ö. Girit contributed equally to this work.

[1] B. D. Josephson, Possible New Effects in Superconductive Tunnelling, Phys. Lett. 1, 251 (1962).

[2] C. W. J. Beenakker, Universal Limit of Critical-Current Fluctuations in Mesoscopic Josephson Junctions, Phys. Rev. Lett. 67, 3836 (1991).

[3] Philip F. Bagwell, Suppression of the Josephson Current through a Narrow, Mesoscopic, Semiconductor Channel by a Single Impurity, Phys. Rev. B 46, 12573 (1992).

[4] L. Bretheau, Ç. Ö. Girit, H. Pothier, D. Esteve, and C. Urbina, Exciting Andreev Pairs in a Superconducting Atomic Contact, Nature (London) 499, 312 (2013).

[5] L. Bretheau, Ph.D. thesis, Ecole Polytechnique, 2012, available online at http://pastel.archives-ouvertes.fr/ pastel-00862029.

[6] F. S. Bergeret, P. Virtanen, T. T. Heikkilä, and J. C. Cuevas, Theory of Microwave-Assisted Supercurrent in Quantum Point Contacts, Phys. Rev. Lett. 105, 117001 (2010).

[7] F. S. Bergeret, P. Virtanen, A. Ozaeta, T. T. Heikkilä, and J.C. Cuevas, Supercurrent and Andreev Bound State Dynamics in Superconducting Quantum Point Contacts under Microwave Irradiation, Phys. Rev. B 84, 054504 (2011).

[8] F. Kos, S.E. Nigg, and L. I. Glazman, FrequencyDependent Admittance of a Short Superconducting Weak Link, Phys. Rev. B 87, 174521 (2013). 
[9] Michel H. Devoret, Daniel Esteve, John M. Martinis, Andrew Cleland, and John Clarke, Resonant Activation of a Brownian Particle out of a Potential Well: Microwave-Enhanced Escape from the Zero-Voltage State of a Josephson Junction, Phys. Rev. B 36, 58 (1987).

[10] M. Fuechsle, J. Bentner, D. A. Ryndyk, M. Reinwald, W. Wegscheider, and C. Strunk, Effect of Microwaves on the Current-Phase Relation of Superconductor-NormalMetal-Superconductor Josephson Junctions, Phys. Rev. Lett. 102, 127001 (2009).

[11] J. J. A. Baselmans, A. F. Morpurgo, B. J. van Wees, and T.M. Klapwijk, Reversing the Direction of the Supercurrent in a Controllable Josephson Junction, Nature (London) 397, 43 (1999).

[12] B. Dassonneville, M. Ferrier, S. Guéron, and H. Bouchiat, Dissipation and Supercurrent Fluctuations in a Diffusive Normal-Metal-Superconductor Ring, Phys. Rev. Lett. 110, 217001 (2013).

[13] Such junctions, which have a large number of Andreev levels, are described quasiclassically in terms of a continuum of subgap states and a continuous occupation function.

[14] M. Zgirski, L. Bretheau, Q. Le Masne, H. Pothier, D. Esteve, and C. Urbina, Evidence for Long-Lived Quasiparticles Trapped in Superconducting Point Contacts, Phys. Rev. Lett. 106, 257003 (2011).

[15] J.M. van Ruitenbeek, A. Alvarez, I. Piñeyro, C. Grahmann, P. Joyez, M. H. Devoret, D. Esteve, and C. Urbina, Adjustable Nanofabricated Atomic Size Contacts, Rev. Sci. Instrum. 67, 108 (1996).

[16] M. L. Della Rocca, M. Chauvin, B. Huard, H. Pothier, D. Esteve, and C. Urbina, Measurement of the Current-Phase Relation of Superconducting Atomic Contacts, Phys. Rev. Lett. 99, 127005 (2007).

[17] D. Vion, A. Aassime, A. Cottet, P. Joyez, H. Pothier, C. Urbina, D. Esteve, and M. H. Devoret, Manipulating the
Quantum State of an Electrical Circuit, Science 296, 886 (2002).

[18] See Supplemental Material at http://link.aps.org/ supplemental/10.1103/PhysRevX.3.041034 for measurements of the current-phase relation, a discussion of the visibility of the transition lines, and data on the dynamics of the occupation of the Andreev level.

[19] Measurements on another contact showed that this structure shifted to lower voltages when longer measurement pulses were applied. This is compatible with the current dependence of the plasma frequency $\nu_{p} \propto\left(1-I_{b} / I_{0}\right)^{1 / 4}$ since switching occurs at smaller values of $I_{b}$ when longer pulses are used.

[20] Transitions in the opposite direction are not represented because their rates are much smaller [14,21].

[21] D. G. Olivares, A. Levy Yeyati, L. Bretheau, Ç. Ö. Girit, H. Pothier, and C. Urbina, Dynamics of Quasiparticle Trapping in Andreev Levels, arXiv:1309.7283.

[22] Moreover, when $0.55 \pi<\varphi<1.15 \pi, 2 E_{A 1}(\varphi+\gamma)$ crosses the plasma energy $h \nu_{p}\left(I_{s w}\right)$ during the rise time of the pulse. Since the excited Andreev state and the plasma mode are strongly coupled [4], and since the lifetime of the plasma mode is in the ns range, this crossing causes relaxation to the ground state.

[23] However, numerical evaluations of the rate for this unpoisoning process using Ref. [8] are an order of magnitude too small to validate this hypothesis.

[24] J-D. Pillet, C. H. L. Quay, P. Morfin, C. Bena, A. Levy Yeyati, and P. Joyez, Andreev Bound States in Supercurrent-Carrying Carbon Nanotubes Revealed, Nat. Phys. 6, 965 (2010).

[25] N. M. Chtchelkatchev and Yu. V. Nazarov, Andreev Quantum Dots for Spin Manipulation, Phys. Rev. Lett. 90, 226806 (2003).

[26] C. Padurariu and Yu. V. Nazarov, Spin Blockade Qubit in a Superconducting Junction, Europhys. Lett. 100, 57006 (2012). 